\title{
Antimicrobial peptides from arachnid venoms and their microbicidal activity in the presence of commercial antibiotics
}

\author{
Francia Garcia ${ }^{1}$, Elba Villegas ${ }^{2}$, Gerardo Pavel Espino-Solis ${ }^{1}$, Alexis Rodriguez ${ }^{1}$, Jorge F Paniagua-Solis ${ }^{3,4}$, \\ Gabriel Sandoval-Lopez ${ }^{3}$, Lourival D Possani ${ }^{1}$ and Gerardo Corzo ${ }^{1}$
}

Two antimicrobial peptides (AMPs), named La47 and Css54, were isolated from the venom of the spider Lachesana sp. and from the scorpion Centruroides suffusus suffusus, respectively. The primary structures of both La47 and Css54 were determined using $\mathrm{N}$-terminal sequencing and mass spectrometry. La47 is identical to the AMP latarcin 3a obtained previously from the venom of the spider Lachesana tarabaevi, but the primary structure of Css54 is unique having $60 \%$ identities to the AMP ponericin-W2 from the venom of the ant Pachycondyla goeldii. Both La47 and Css54 have typical $\alpha$-helix secondary structures in hydrophobic mimicking environments. The biological activities of both La47 and Css54 were compared with the AMP Pin2 isolated from the venom of the scorpion Pandinus imperator. La47 has lower antimicrobial and hemolytic activities compared with Css54 and Pin2. In addition, La47 and Pin2 were evaluated in the presence of the commercial antibiotics, chloramphenicol, ampicillin, novobiocin, streptomycin and kanamycin. Interestingly, the best antimicrobial combinations were obtained with mixtures of La47 and Pin2 with the antibiotics chloramphenicol, streptomycin and kanamycin, respectively. Furthermore, the novel peptide Css54 was evaluated in the presence of antibiotics used for the treatment of tuberculosis, isoniazid, rifampicin, pyrazinamide and ethambutol. Although the mixtures of Css54 with isoniazid, pyrazinamide or ethambutol inhibit the growth of Staphylococcus aureus, the best effect was found with rifampicin. Overall, these data show a motivating outlook for potential clinical treatments of bacterial infections using AMPs and commercial antibiotics.

The Journal of Antibiotics (2013) 66, 3-10; doi:10.1038/ja.2012.87; published online 24 October 2012

Keywords: antimicrobial peptide; peptide; scorpion; spider

\section{INTRODUCTION}

Since the development of penicillin in the 1940s, the synthesis and use of different antibiotics have had an important impact on human health. The emergence of resistant strains has made bacterial infections increasingly difficult to treat with available antibiotics. Particularly, bacteria have developed different mechanisms for acquiring or modifying their genes, an accelerate mechanism of adaptation for survival of both pathogenic and non-pathogenic microorganisms. ${ }^{1,2}$ Currently, all the infection-controlling drugs on the market or in company's pipeline development are those related to traditional antibiotic model. New genomic technologies have, so far, failed to deliver novel therapeutics, with only two new classes of antibiotics: the oxazolidinones and lipopeptides entering in the market in the last 30 years. $^{3}$ In this way, the antibiotic resistance problem have motivated the research on the discovery of new drugs for bacterial infection control, such as finding novel non-traditional antibiotic sources or elaborating combinations between commercial available antibiotics to generate synergistic effects. ${ }^{4,5}$

On the other hand, amphipathic peptides with antimicrobial activity are widely distributed throughout nature and produced in species of the kingdoms Monera (for example, Eubacteria), Protista (for example, protozoans and algae), Fungi (for example, yeasts), Plantae (plants) and Animalia (for example, insects, fish, amphibians, reptiles, birds and mammals). ${ }^{6}$ These antimicrobial peptides (AMPs) are small molecules with wide variations in their mass, amino-acid residue composition, charge, three-dimensional structure and biological characteristics. ${ }^{7}$ In some species these peptides serve as the primary antimicrobial defense mechanism, yet in other species they serve as an adjunct to existing innate and adaptive immune systems. ${ }^{8}$

\footnotetext{
${ }^{1}$ Departamento de Medicina Molecular y Bioprocesos, Instituto de Biotecnología, Universidad Nacional Autónoma de México, Apartado Postal 510-3, Cuernavaca Mor, México; ${ }^{2}$ Centro de Investigación en Biotecnología, Universidad Autónoma del Estado de Morelos, Avenida Universidad 2001, Cuernavaca, Morelos, México and ${ }^{3}$ Laboratorios Silanes S.A. de C.V., Amores 1304, Col. Del Valle, México.

${ }^{4}$ Current address: Teraclón IDF, S.L., Capitán Haya 38, 28020, Madrid, España.

Correspondence: Dr G Corzo, Departamento de Medicina Molecular y Bioprocesos, Instituto de Biotecnología, Universidad Nacional Autónoma de México, UNAM, Apartado Postal 510-3, Cuernavaca, Morelos 62210, México.

E-mail: corzo@ibt.unam.mx

Received 31 May 2012; revised 10 September 2012; accepted 20 September 2012; published online 24 October 2012
} 
Spider and scorpion venoms contain AMPs, which permeate lipid membranes through formation of pores. ${ }^{9-17}$ The accepted mechanism of action of these pore-forming peptides is by the disruption of the bacterial membranes by a membrane pore-formation or by a membrane detergent-like effect. ${ }^{18,19}$ Nevertheless, their mechanism of binding to lipid membranes differs from one to another depending on their amino-acid sequence, size, type of structure formation, charge, hydrophobicity and amphipathicity. ${ }^{18-20}$ Although the main purpose of pore-forming peptides found in the venoms of arachnids is to depolarize the cell membrane and tissues of their prey, their antibacterial activity probably has the function of a bona fide antibiotic that is useful for the spider or scorpion to clean their biological conducts that carry venom from the venom gland to the tip of the scorpion stinger or to the spider fangs. ${ }^{17}$

The ability of microbes to rapidly evolve resistance compromises the efficacy of antibiotics, necessitating the continuous discovery of structurally novel natural products with antimicrobial properties. Being aware of the antibiotic resistance problem we start to explore into new molecules from the arachnid venoms source. Therefore, the aim of this work was to search for unknown novel antimicrobial agents in the venoms of two arachnids, the spider Lachesana sp. and the scorpion Centruroides suffusus suffusus (C.s. suffusus), and to test their antimicrobial effects in the presence of commercial antibiotics, expecting that this strategy would improve their antimicrobial efficacy.

\section{MATERIALS AND METHODS}

\section{Biologicals}

The scorpions were collected from the state of Durango in Mexico and maintained alive in good conditions following the Mexican official requirements and licenses (SEMARNAT FAUT-0184 and MOR-IN-166-0704) C. s. suffusus crude venom was obtained by electrical stimulation and was frozen and stored at $-20^{\circ} \mathrm{C}$ until use. The lyophilized venom from Lachesana sp. was purchased from SpiderPharm (Yarnell, AZ, USA). The microbial strains Escherichia coli (ATCC 25922) and Staphylococcus aureus (ATCC 25923) were purchased from the American Type Culture Collection (ATCC, Manassas, VA, USA) through The Global Bioresource Center by UNAM. The AMP Pin2, used as positive control, was synthesized according to Corzo et al. ${ }^{10}$ Endoproteinase Glu-C (E.C.3.4.21.19) and Endoproteinase Lys-C (E.C.3.4.21.4) were purchased from Roche (Nutley, NJ, USA). The antibiotics chloramphenicol, ampicillin, novobiocin, streptomycin and kanamycin were from Sigma (Mexico City, Mexico). The antibiotics isoniazid, rifampicin, pyrazinamide and ethambutol were donated by Laboratorios Silanes SA de CV (Mexico City, Mexico)

\section{Isolation of AMPs from arachnid venoms}

C. s. suffusus soluble venom ( $10 \mathrm{mg}$ ) was dissolved in $1 \mathrm{ml}$ of $0.1 \%$ aqueous trifluoroacetic acid (TFA) containing 5\% acetonitrile, and the insoluble material was removed by centrifugation at $14000 \mathrm{~g}$ for $5 \mathrm{~min}$. The supernatant was filtered through Millex-GV filters $(0.45 \mu \mathrm{m})$ before fractionation. Diluted venom was first fractionated using a reverse phase semipreparative $\mathrm{C}_{18}$ column $\left(5 \mathrm{C}_{18} \mathrm{MS}, 10 \times 250 \mathrm{~mm}\right.$, Nacalai Tesque, Japan) equilibrated in $0.1 \%$ TFA in water (solution A). A linear gradient from solution A to $60 \%$ solution $\mathrm{B}$ $(0.1 \%$ TFA in acetonitrile) was run for $60 \mathrm{~min}$, at a flow rate of $2 \mathrm{ml}$ per min. Effluent absorbance was monitored at $230 \mathrm{~nm}$. Fractions with antimicrobial activity were finally purified using a reverse phase analytical $\mathrm{C}_{18}$ column $(4.6 \times 250 \mathrm{~mm}$, Nacalai Tesque, Japan) using the same gradient system as above, with a flow rate of $1 \mathrm{ml}$ per min. The same procedure was used for fractionation of Lachesana sp. soluble venom $(10 \mathrm{mg})$

\section{Antimicrobial assays}

The isolation of the two AMPs, La47 and Css54, from the soluble venoms of Lachesana sp. and C. s. suffusus, respectively, was done following plate growth inhibition of E. coli and S. aureus in the presence of the already fractioned arachnid venom samples. Briefly, vacuum-dried high pressure liquid chromatography (HPLC) fractions were dissolved in $20 \mu \mathrm{l}$ of distilled water. The bacteria E. coli and S. aureus were grown in Mueller-Hinton broth medium (Sigma-Aldrich, St Louis, MO, USA) for $8 \mathrm{~h}$. An aliquot of $0.1 \mathrm{ml}$ of bacterial suspension (optical density $(\mathrm{OD})_{595 \mathrm{~nm}}=0.3-0.8$ ) was added to $9.9 \mathrm{ml}$ of sterile Mueller-Hinton broth medium to make a 1:100 dilution. One milliliter of the dilution was added to $9 \mathrm{ml}$ of warm $\left(\sim 45^{\circ} \mathrm{C}\right)$ Mueller-Hinton agar medium, and the medium was poured into $100 \times 20 \mathrm{~mm}^{2}$ sterile petri dishes. Bacterial count, depending on the strain, was $c a 10^{6}-10^{7}$ colony-forming units $\mathrm{ml}^{-1}$. Then, $5 \mu \mathrm{l}$ of each resuspended HPLC fractions were applied to the plate surface. The plates were incubated at $37^{\circ} \mathrm{C}$ for $12-14 \mathrm{~h}$ and growth inhibition was detected as clear spots on the plate surface.

Growth inhibition curves in Mueller-Hinton broth were obtained using pure AMPs, antibiotics and mixtures of AMPs with antibiotics. Briefly, the inoculums were prepared from fresh bacterial cultures of either $E$. coli or S. aureus. Serial dilutions of either AMPs or commercial antibiotics were made at different concentrations. For growth curves, the final volume in each vial was $200 \mu \mathrm{l}$ and the cell count was $\sim 1 \times 10^{4} \mathrm{CFU} \mathrm{ml}^{-1}$ for each of the strains. Serial dilutions of peptides were made, and an aliquot of cell suspension was added to each vial. The OD of each vial was measured at $595 \mathrm{~nm}$ in an ELISA reader (Bio-Rad, model 450, Hercules, CA, USA). The positive contro contained only the bacterial suspension, and the negative control contained only culture medium.

\section{Enzymatic digestions of peptides}

The AMPs La47 and Css54 were subjected to enzymatic hydrolysis. Lys-C hydrolysis was carried out in $0.1 \mathrm{~m}$ sodium bicarbonate buffer $(\mathrm{pH} 8.1)$ at $37^{\circ} \mathrm{C}$ for $3 \mathrm{~h}$, using a 1:50 (w/w) enzyme to substrate ratio. Hydrolysis with type XVII-B endoproteinase Glu-C from S. aureus V8 was carried out in $0.1 \mathrm{M}$ sodium bicarbonate buffer $(\mathrm{pH} 7.6)$, at $37^{\circ} \mathrm{C}$ for $3 \mathrm{~h}$, using a $1: 20(\mathrm{w} / \mathrm{w})$ enzyme to substrate ratio. The Lys- $\mathrm{C}$ digest was fractionated by reverse phase HPLC using a $\mathrm{C}_{18}$ column $(4.6 \times 250 \mathrm{~mm}$, Nacalai Tesque, Japan $)$ and run using a linear gradient from solution A to $60 \%$ solution B (see above) for $60 \mathrm{~min}$ at the flow rate of $1 \mathrm{ml}$ per min. Effluent absorbance was monitored at $230 \mathrm{~nm}$. The Lys-C fractions were vacuum dried, resuspended, analyzed by ESI-MS and N-terminal sequenced. The endoproteinase Glu-C digest was also fractionated using the same conditions with the Lys-C fragments.

\section{Sequence analysis and mass spectrometry}

The AMPs and their enzymatic fragments were sequenced from their $\mathrm{N}$-terminal by Edman degradation using a LF3000 Protein Sequencer (Beckman, CA, USA). The mass identity of all isolated peptides and the peptide fragments from the enzymatic cleavage was verified by ESI-MS using a Finnigan $\mathrm{LCQ}^{\mathrm{DUO}}$ ion trap mass spectrometer (San José, CA, USA). MS/MS spectra were obtained on a Brucker Daltonics Ultraflex MALDI TOF-TOF spectrometer (Brucker Daltonics, Billerica, MA, USA). MALDI experiments were performed using $\alpha$-cyano-4-hydroxycinnamic acid (Aldrich) as the matrix.

\section{Peptide synthesis and purification}

La47 and Css54 were chemically synthesized by a solid-phase method using the Fmoc methodology at the GenScript (San Diego, CA, USA). The crude synthetic peptide was dissolved in 30\% aqueous acetonitrile solution and separated by reverse phase HPLC on a semipreparative $\mathrm{C}_{18}$ column $\left(10 \times 250 \mathrm{~mm}\right.$, Nacalai Tesque, Japan). The $\mathrm{C}_{18}$ column was equilibrated in $30 \%$ aqueous acetonitrile containing $0.1 \%$ TFA. The synthetic peptides were purified using a linear gradient from 30 to $60 \%$ solution B (see above) for $30 \mathrm{~min}$, at a flow rate of $1 \mathrm{ml}$ per min. Effluent absorbance was monitored at $230 \mathrm{~nm}$. The structural identity between synthetic and natural peptides was either verified by co-elution or by similar retention time experiments using reverse phase chromatography. The mass identity between synthetic and natural peptides was verified by ESI-MS mass spectrometry.

\section{Red blood cell hemolysis assays}

Hemolytical activity was determined by incubating a 10\% (v/v) suspension of human red blood cells with selected peptides. Blood cells were rinsed several times in PBS (phosphate-buffered saline) by centrifugation for $3 \mathrm{~min}$ at $3000 \mathrm{~g}$ 
until the OD of the supernatant reached the OD of the control (PBS only). Red blood cells were then incubated at room temperature for $1 \mathrm{~h}$ in deionized water (positive control), in PBS (blank), or with the appropriate amount of AMPs. The samples were then centrifuged at $4000 \mathrm{~g}$ for $5 \mathrm{~min}$, the supernatant was separated from the pellet, and its absorbance measured at $570 \mathrm{~nm}$. The relative OD compared with that of the suspension treated with deionized water defined the percentage of hemolysis.

\section{Secondary structure analysis}

Circular dichroism spectra were obtained on a Jasco J-725 spectropolarimeter (Jasco, Tokyo, Japan). The spectra were measured from 260 to $190 \mathrm{~nm}$ in $60 \%$ trifluoroethanol to promote hydrogen-bonding, ${ }^{17,21} \mathrm{pH} 7.1$ at room temperature, with a $1-\mathrm{mm}$ path-length cell. Data were collected at $1 \mathrm{~nm}$ with a scan rate of $50 \mathrm{~nm}$ per min and a time constant of $1 \mathrm{~s}$. The concentration of the peptides was 100 and $50 \mu \mathrm{g} \mathrm{ml}^{-1}$ of La47 and Css54, respectively. Data were the average of two separate recordings.

\section{Antibiotic checkerboard analysis}

The combinations tested (in duplicates) against each bacterium were La47 and Pin2 against E. coli and S. aureus, respectively, plus the commercial antibiotics chloramphenicol, ampicillin, novobiocin, streptomycin or kanamycin. In addition, Css54 was tested against $S$. aureus in the presence of isoniazid, rifampicin, pyrazinamide or ethambutol. The concentration range of each antibiotic in combination with AMP ranged from 1/32 times the minimal inhibitory concentration (MIC) $(1 / 32 \times$ MIC) to 4 MIC. Dilutons of Antibiotics were made manually with twofold dilutions. The initial inoculum was prepared as described above. To evaluate the effect of the combinations, the fractional inhibitory concentration (FIC) was calculated for each antibiotic in each combination. ${ }^{22,23}$ The following formulas were used to calculate the FIC index: FIC of AMP = MIC of AMP in combination with Antibiotic/MIC of AMP alone, FIC of Antibiotic = MIC of Antibiotic in combination with AMP/MIC of Antibiotic alone, and FIC index $=$ FIC of AMP + FIC of Antibiotic. Synergy was defined as an FIC index of $<1$. Additive was defined as an FIC index of between $>1$ and $<4$. Antagonism was defined as an FIC index of $>4$.

\section{Statistical analysis}

The last significant difference method was used to determine whether statistically significant differences occurred among the mean values obtained. The Hemolysis Constants $\left(\mathrm{IC}_{50}\right)$ were obtained using a non-linear regression where the data fit the Boltzmann sigmoidal equation using the software package Prism 4 (GraphPad, La Jolla, CA, USA).

\section{RESULTS}

\section{Isolation and primary structure analysis}

The crude venoms of Lachesana sp. and C. s. suffusus were separated by reverse phase HPLC (Figure 1). Sixty chromatographic fractions from either spider or scorpion venoms were manually collected, vacuum dried and then dissolved in milliQ water. Further, all fractions were assayed for antibacterial activity against E. coli and S. aureus. The chromatographic fractions named La47 and Css54 from Lachesana sp. and C. s. suffusus, respectively (Figure 1), gave positive results against E. coli and S. aureus). These two fractions were further analyzed by a second chromatographic step using an analytical $\mathrm{C}_{18}$ reverse phase column. The protein fractions preserving their antibiotic properties were also labeled La47 and Css54, respectively (Figure 1, insets). The peptides, La47 and Css54, were obtained pure and did not contain disulfide bridges because their molecular masses did not change after reduction and alkylation of the cystines (data not shown). The final protein recovery yield of La47 and Css54 was 35 and $115 \mu \mathrm{g}$ from $10 \mathrm{mg}$ initial material used; that is, 0.35 and $1.15 \%$ of their whole soluble venom, respectively. La47 and Css54 were $\mathrm{N}$-terminal sequenced by Edman degradation, which allowed the identification of their first 20 and 18 amino-acid residues, respectively
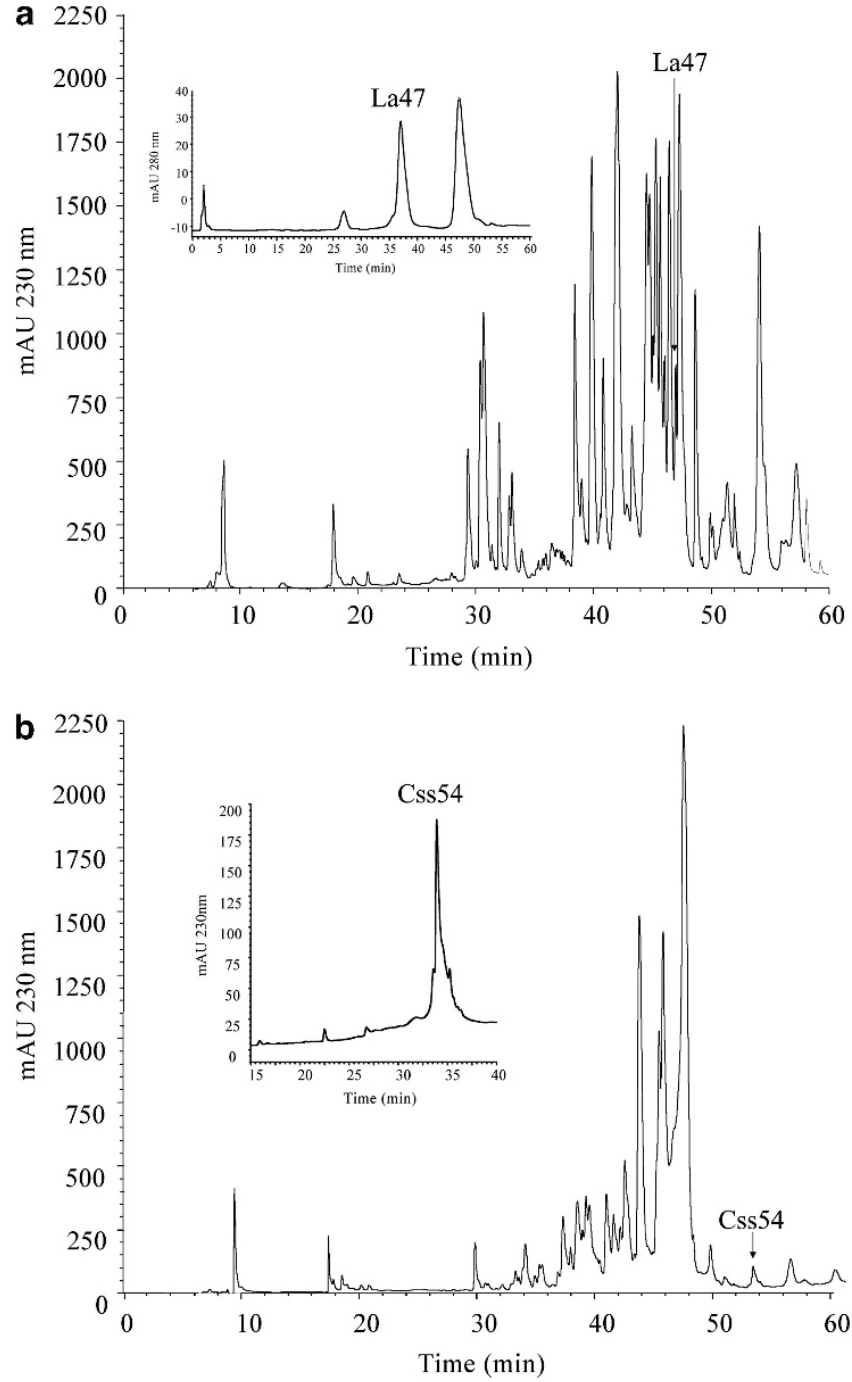

Figure 1 HPLC separation of two arachnid venoms. The venom from the spider Lachesana sp. (a) and that from the scorpion C. s. suffusus (b) were separated using a semi-preparative reverse phase $\mathrm{C}_{18}$ column, at a flow rate of $2 \mathrm{ml}$ per min, with linear gradients from 0 to $60 \% \mathrm{~B}$ in $60 \mathrm{~min}$. The inset figures represent the second step of purification showing the isolation of La47 and Css54 using an analytical reverse phase $\mathrm{C}_{18}$ column, at a flow rate of $1 \mathrm{ml}$ per min, with linear gradients from 10 to $60 \% \mathrm{~B}$ in $50 \mathrm{~min}$ and from 20 to $60 \%$ B in $40 \mathrm{~min}$, respectively. The peptide fractions containing either La47 or Css54 are labeled.

Table 1 Amino-acid sequences and molecular masses of La5 and Css54

\begin{tabular}{|c|c|c|c|}
\hline Peptide & Sequence & $\mathrm{M}_{w} \mathrm{Cal}(\mathrm{Da})$ & $\mathrm{M}_{w} \operatorname{Exp}(D a)$ \\
\hline La5 & $\begin{array}{l}\text { SWKSMAKKLKEYMEKLKQRA } \\
<\longrightarrow<\text { Direct } \\
<\longrightarrow<->\text { Glu-C }\end{array}$ & $2,484.0$ & $2,484.9$ \\
\hline Css54 & $\begin{array}{l}\text { FFGSLLSLGSKLLPSVFKLFQRKKE } \\
\begin{aligned}<->\text { Direct } \\
<\longrightarrow<-><->\text { LyS }-\mathrm{C} \\
<->\text { MS } / \text { MS }\end{aligned}\end{array}$ & $2,870.4$ & $2,870.4$ \\
\hline
\end{tabular}

$M_{\mathrm{w}}$ Cal, means calculated molecular mass.

$M_{\mathrm{w}}$ Exp, means experimental molecular mass. 

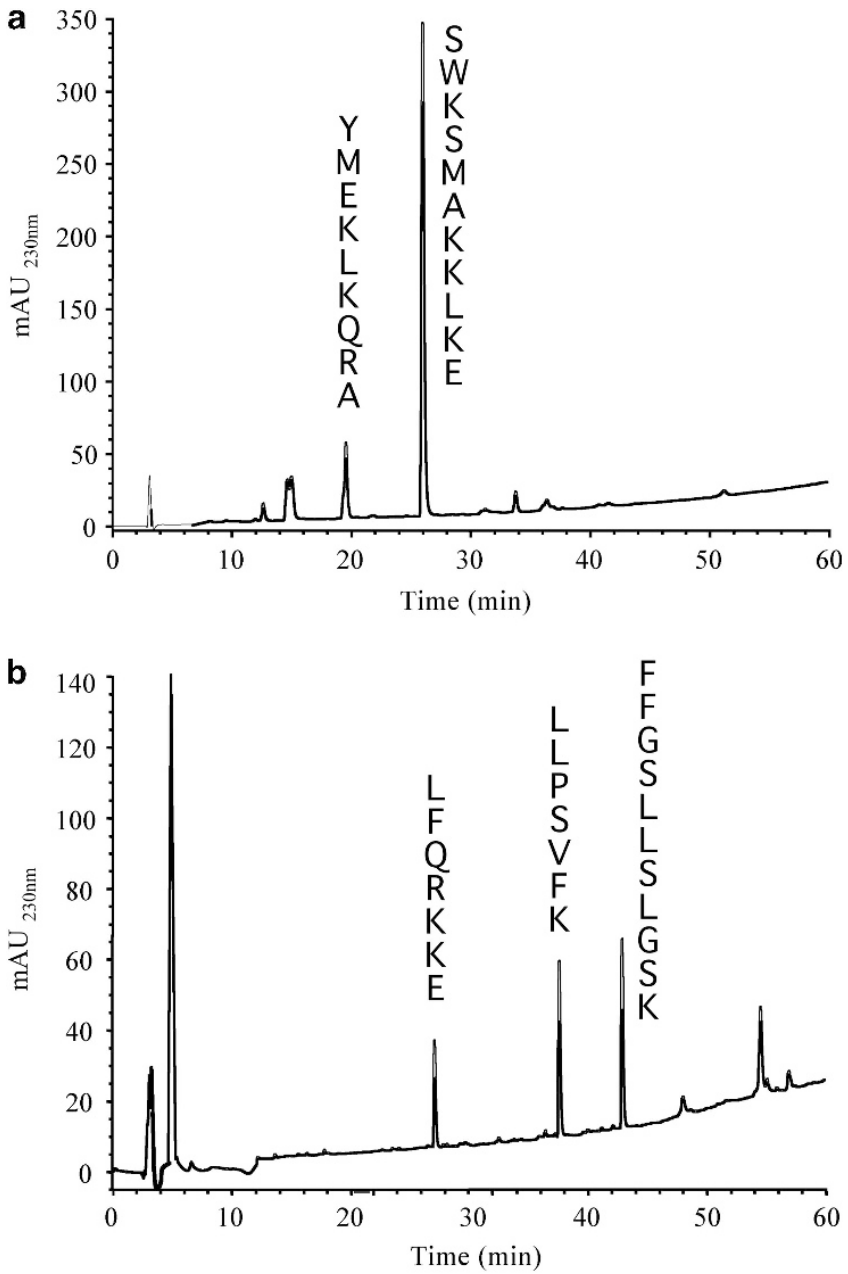

Figure 2 Enzymatic digestion of La47 and Css54. The peptide fragments from the enzymatic digestion of La47 (a) and Css54 (b) were separated using an analytical reverse phase $\mathrm{C}_{18}$ column, at a flow rate of $1 \mathrm{ml}$ per $\mathrm{min}$, with linear gradients from 0 to $60 \% \mathrm{~B}$ in $60 \mathrm{~min}$. The amino-acid sequences of each fraction were obtained by automatic $\mathrm{N}$-terminal aminoacid sequencing.

(Table 1). La47 was further digested by Glu-C, and two peptide fragments were obtained after separation by reverse phase HPLC (Figure 2a). Amino-acid sequencing of these two peptide fractions permitted the identification of the full primary structure of La47, where the overlapping of the two segments were confirmed by the previous $\mathrm{N}$-terminal sequence up to residue number 18 (Table 1). Likewise, the AMP Css54 was enzymatically digested with Lys-C, and three peptide fragments were obtained after separation by reverse phase HPLC (Figure $2 \mathrm{~b}$ ). The N-terminal sequencing of two peptide fragments from Css54 confirmed its first 20 residues (Table 1). Additionally, because of the low amounts obtained of the third enzymatic fragment, the amino-acid sequence of this fragment corresponding to the C-terminal residues was determined by MS/ MS given the final sequence LFQRKKE, which overlaps with the direct sequence obtained with the native peptide. La47 and Css54 have a calculated molecular mass of 2484.0 and $2870.4 \mathrm{Da}$, respectively, that agrees with the mass found by mass spectrometry analysis of 2484.9 and $2870.4 \mathrm{Da}$ (Table 1).

La47 was identical (100\%) to the AMP latarcin 3a from the venom of the spider Lachesana tarabaevi. ${ }^{24}$ In addition, within the same
Table 2 Sequence alignment of La47 and Css54 with amphipathic antimicrobials from other animal species.

\begin{tabular}{|c|c|c|c|}
\hline Peptide & Sequence & $\begin{array}{l}\text { Identity to } \\
\text { La47 (\%) }\end{array}$ & $\begin{array}{l}\text { Accession } \\
\text { number }\end{array}$ \\
\hline La47 & SWKSMAKKLKEYMEKLKQRA & & \\
\hline Ltc $3 a$ & SWKSMAKKLKEYMEKLKQRA & 100 & Q1ELU3 \\
\hline Ltc $3 b$ & $\begin{array}{l}\text { SWASMAKKLKEYMEKLKQRA } \\
* * * * * * * * * * * * * * * * * * *\end{array}$ & 95 & Q1ELU2 \\
\hline Peptide & Sequence & $\begin{array}{l}\text { Identity to } \\
\text { Css54 (\%) }\end{array}$ & $\begin{array}{l}\text { Accession } \\
\text { number }\end{array}$ \\
\hline Css54 & FFGSLLSLGSKLLPSVFKLFQRKKE & & \\
\hline Ponericin-W2 & WLGSALKIGAKLLPSVVGLFQKKKK & 60 & P82424 \\
\hline Ponericin-W1 & WLGSALKIGAKLLPSVVGLFKKKKQ & 56 & P82423 \\
\hline Ponericin-W5 & FWGALIKGAAKLIPSVVGLFKKKQ & 45 & P82427 \\
\hline Pandinin2 & FWGALAKGALKLIPSLFSSFSKKD & 41 & P83240 \\
\hline Gaegurin5 & FLGALFKVASKVLPSVKCAITKKC & 37 & P80399 \\
\hline Brevinin1 & FLPVLAGIAAKVVPALFCKITKKC & 25 & P32423 \\
\hline Pipinin 1 & FLPIIAGVAAKVFPKIFCAISKKC & 20 & P82841 \\
\hline Pipinin2 & $\begin{array}{c}\text { FLPIIAGIAAKVFPKIFCAISKKC } \\
\star *\end{array}$ & 20 & Q8QFQ5 \\
\hline
\end{tabular}

The sequence alignment was done with the Divide-and-Conquer Multiple Sequence Alignment program using the PAM 250 from http://bibiserv.TechFak.Uni-Bielefeld.DE/dca/. For clarity, the order of the sequences was set according to their percentage of identity, where the percentage of sequence identity based on PAM250 is given relative to La47 or Css54. The symbols '*', ' $:$ ' and '.' have been introduced to show identical, very conservative and conservative residues, respectively. Toxin sequences references are databank accession numbers from either UnitProt or GenBank as retrieved from www.ncbi.nih.gov/entrez.

spider venom there is a second AMP variant named latarcin $3 \mathrm{~b}$, with a single residue modification (Table 2). Although the primary structure of La47 has been revealed previously, the separation procedures shown in this report is different to the described one; thus, it contributes with new data to this work. ${ }^{24}$ Likewise, the biological activities of latarcin 3 a (La47) on E. coli, S. aureus and human red blood cells have not been reported previously. Contrary to La47, the primary structure of Css54 is unique and shows some identity to the AMPs Ponericin-W2 (60\%), Pin2 (41\%), Gaegurin-5 (37\%) and Brevenin-1 (25\%) isolated from the venoms of the South American ant Pachycondyla goeldii, ${ }^{25}$ and the African scorpion Pandinus imperator, and from the skin of the Asian amphibian Rana rugosa ${ }^{26}$ and Rana brevipoda porsa, ${ }^{27}$ respectively (Table 2).

\section{Biological activities and structural features}

The MICs in agar media of La47 and Css54 were compared with that of Pin2, a potent bactericide isolated from the venom of the African scorpion P. imperator. ${ }^{10}$ The antibacterial activity of Pin 2 was higher compared with the activity of La47 but similar to Css54 (Table 3). In addition, the antibacterial activity of La47 was less potent than that of Css54 and Pin2 in Mueller-Hinton broth (Figure 3). The concentration of La47 required for inhibition of $S$. aureus growth was $67.2 \mu \mathrm{g} \mathrm{ml}^{-1}$, and Css54 and Pin2 needed 15.4 and $4.2 \mu \mathrm{g} \mathrm{ml}^{-1}$ concentration, respectively. These data confirmed that La47 was the less active peptide in both agar and broth media. Furthermore, since most of the AMPs disrupt the cell membrane potential by poreformation, they may be also toxic to eukaryotic cells. Therefore, in order to determine their cytolytic behavior, the hemolytic activities of La47 and Css54 against human erythrocytes were calculated. Css54 showed higher hemolytic activity than to that of La47. At $25 \mu \mathrm{M}$, the percentage of hemolysis was 100, 83 and $2 \%$ for Pin2, Css 54 and La47, respectively (Figure 4a). On the other hand, the secondary 
Table 3 Minimum inhibitory concentrations of AMPs against $E$. coli and $S$. aureus and commercial antibiotics in Muller-Hilton Agar

\begin{tabular}{lcc}
\hline Antibiotics & MIC $\left(\mu g \mathrm{~m}^{-1}\right)$ E. coli & MIC $\left(\mu g \mathrm{I}^{-1}\right)$ S. aureus \\
\hline La47 & 100 & 84 \\
Css54 & 12.5 & 12.5 \\
Pin2 & 10.4 & 10.4 \\
Ampicillin & 2.5 & 10 \\
Chloramphenicol & 6.2 & 6.2 \\
Streptomycin & 125 & 125 \\
Kanamycin & 250 & 125 \\
Novobiocin & 500 & 500 \\
Ethambutol & ND & 500 \\
Pyrazinamide & ND & 500 \\
Isoniazid & ND & 500 \\
Rifampicin & ND & 0.78 \\
\hline
\end{tabular}

Abbreviation: AMP, antimicrobial peptide.

ND means that minimal inhibitory concentrations (MICs) were not determined.

structure of La47 and Css54 was measured by CD and analyzed using a neural network of $33 \mathrm{CD}$ spectra of the proteins in the range 260 to $200 \mathrm{~nm}$ at $1 \mathrm{~nm}$ intervals. Broadly, they had distinctive negative ellipticities at 208 and $222 \mathrm{~nm}$ in $60 \%$ TFE, which are common of $\alpha$-helix secondary structures (Figure $4 \mathrm{~b}$ ). Therefore, the CD data indicate that both La47 and Css54 most likely adopt a $\alpha$-helix folding pattern.

\section{Antimicrobial activity of AMPs in the presence of commercial antibiotics}

Most findings reported in the literature agree that the consensus mechanism of action of these helical amphipathic cationic AMPs is through pore-formation of cell membranes. Apart from this general agreement, AMPs could also be translocated into the cell cytoplasmic environment and could be exerting their effect by interacting with proteins involved in cell metabolism processes. ${ }^{28}$ Therefore, because of their pore-forming and translocation abilities, these AMPs could improve the access of other molecules from the outside environment into the cell cytoplasm. Specifically, if the cell membrane is a physical barrier for such molecules, the use of AMPs could facilitate the incorporation of antibiotics into the cytoplasm of bacteria cells. To test this hypothesis, increasing concentrations of AMPs in the presence of increasing concentrations of commercial antibiotics were tested against cell cultures of E. coli and $S$. aureus. To reduce the number of experiments, La47 was tested only against E. coli, and Css54 and Pin2 were tested only against $S$. aureus. The selection of commercial antibiotics was based on covering different mechanisms of action. Therefore, the antibiotics used were ampicillin (cell wall), chloramphenicol (ribosomal subunit 50S), streptomycin (ribosomal subunit 30S), kanamycin (ribosomal subunit 30S) and novobiocin (DNA girase). To observe any antagonistic, additive or synergistic effect in detriment of the bacterial growth the FIC index was calculated (see Materials and Methods). Figure 5 shows the improved antibiotic capacity of La47 and Pin2 in the presence of kanamycin. Here, the combination of $50 \mu \mathrm{g} \mathrm{ml}^{-1}$ of La47 together with $15.6 \mu \mathrm{g} \mathrm{m}^{-1}$ of kanamycin inhibits the growth of $E$. coli. The same effect could be obtained with higher $\left(>62.5 \mu \mathrm{g} \mathrm{ml}^{-1}\right)$ concentrations of kanamycin alone. However, the mixture of only $2.8 \mu \mathrm{g} \mathrm{ml}^{-1}$ of Pin2 together with $15.6 \mu \mathrm{g} \mathrm{ml}^{-1}$ of kanamycin was enough to inhibit the growth of S. aureus (Figure 5). The best antimicrobial effects for La47 and Pin2 were observed in the presence of chloramphenicol, streptomycin and
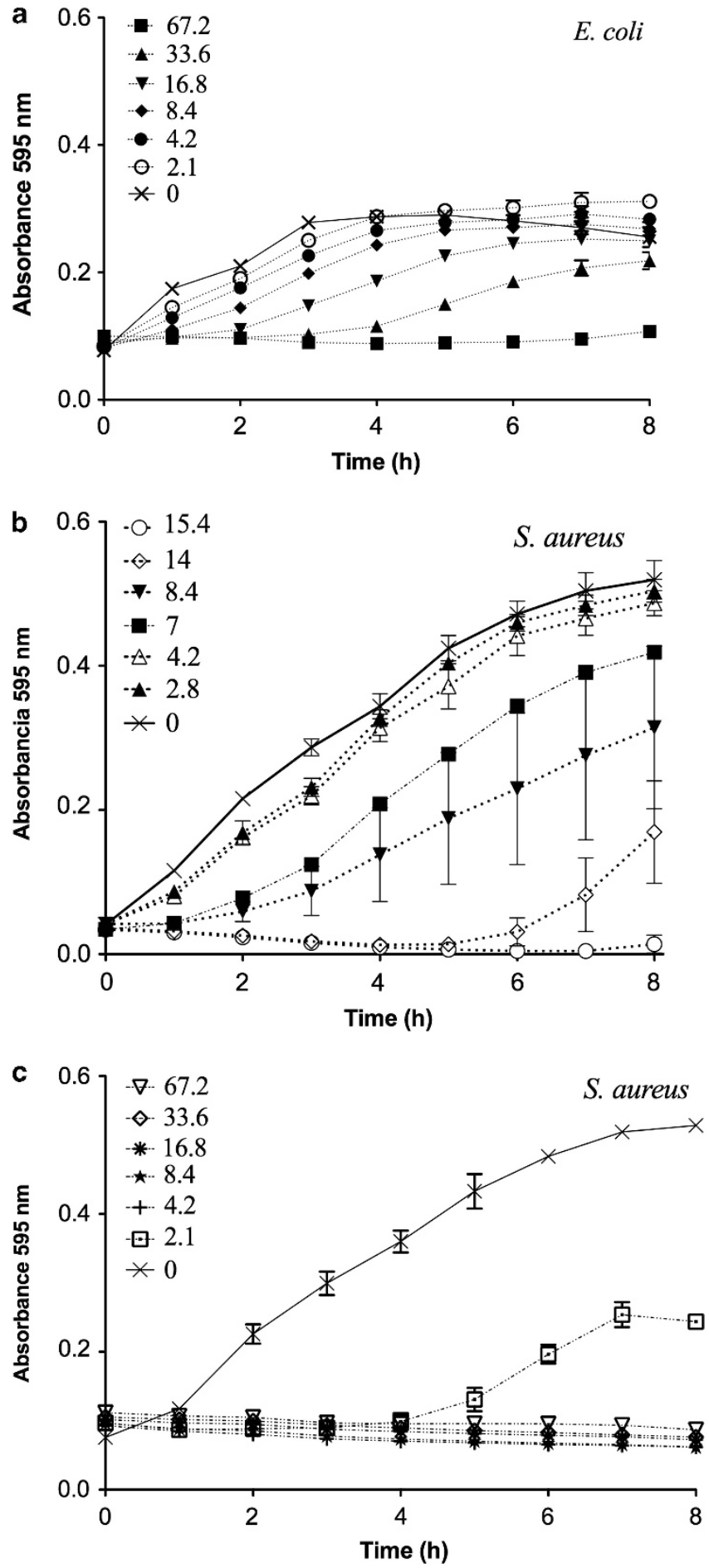

Figure 3 Dose-response activities of AMPs. Antimicrobial activities of La47 $\left(0,2.1,4.2,8.4,16.8 .33 .6\right.$ and $\left.67.2 \mu \mathrm{g} \mathrm{ml}^{-1}\right)$ against E. coli (a), and Css54 (0, 2.8, 4.2, 7, 8.4, 14 and $\left.15.4 \mu \mathrm{g} \mathrm{ml}^{-1}\right)$ and Pin2 (0, 2.1, 4.2, 8.4, 16.8. 33.6 and $67.2 \mu \mathrm{g} \mathrm{ml}^{-1}$ ) against $S$. aureus (b, c, respectively) in Muller Hinton broth.

kanamycin (Table 4). Furthermore, only Css54 was tested in the presence of antibiotics used for the treatment of TB (tuberculosis). The rational to perform these experiments was to use the newly characterized peptide Css54 and to observe if further commercial antibiotics 

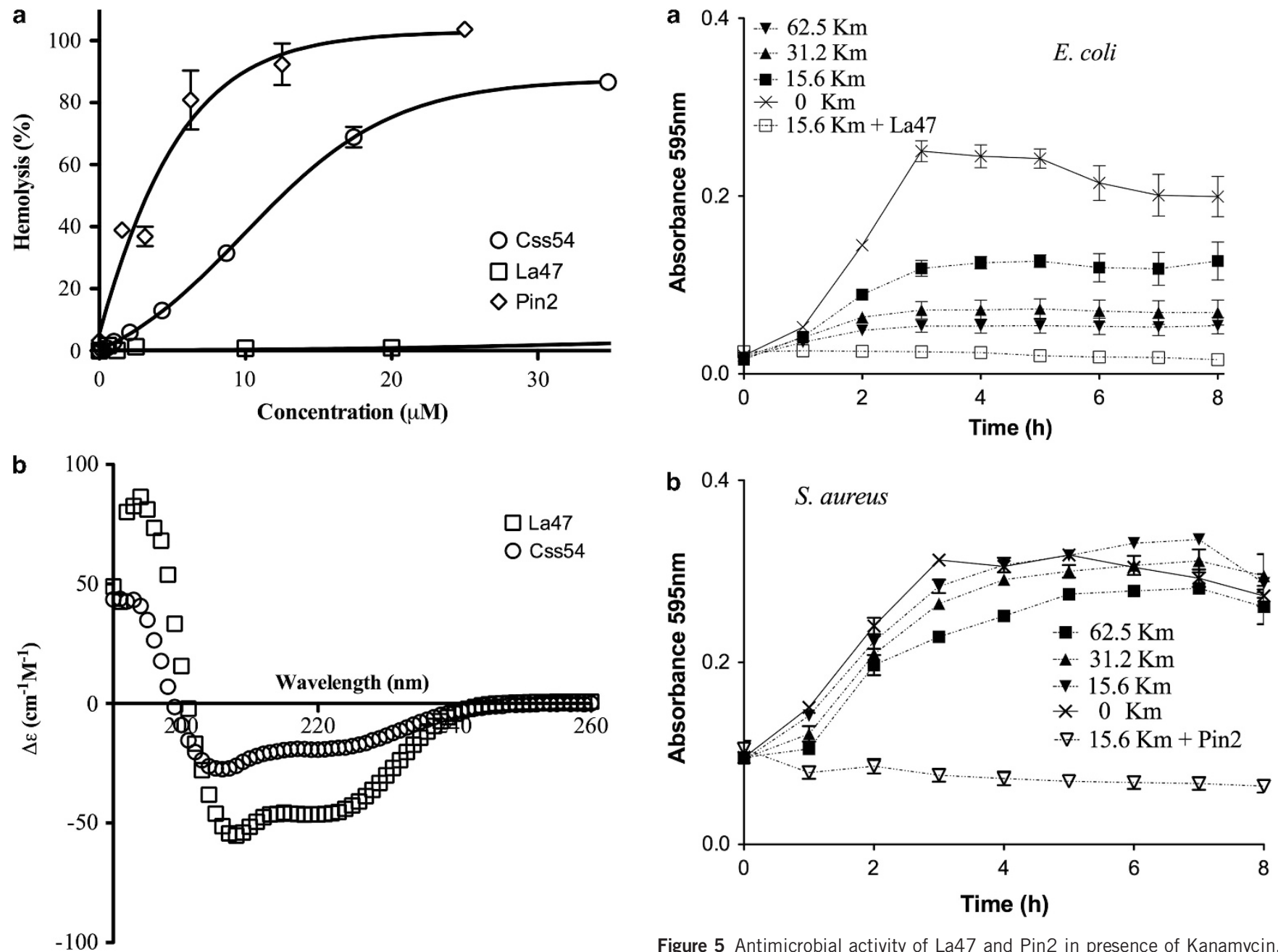

Figure 4 Hemolytic activity and circular dichroism of AMPs. The hemolytic activities were tested in human red blood cells (a). The circular dichroism of La47 and Css54 was performed at 100 and $50 \mu \mathrm{g} \mathrm{ml} \mathrm{I}^{-1}$, respectively (b).

Figure 5 Antimicrobial activity of La47 and Pin2 in presence of Kanamycin. E. coli (a) and S. aureus (b) were grown in presence of Kanamycin (0, 15.6, 31.2 and $62.5 \mu \mathrm{g} \mathrm{m}^{-1}$ ) without the antimicrobial peptides La47 and Pin2. In addition, E. coli (a) and $S$. aureus (b) were also grown in presence of Kanamycin $\left(15.6 \mu \mathrm{g} \mathrm{ml}^{-1}\right)$ in combination with the antimicrobial peptides La47 (Figure 5a, $50 \mu \mathrm{g} \mathrm{ml}^{-1}$ ) and Pin2 (Figure $5 \mathrm{~b}$ ), $2.8 \mu \mathrm{g} \mathrm{ml}^{-1}$ ), respectively.

used for specific pathogens could also be used in combination with AMPs. S. aureus was used as a model because several clinical isolates of this Gram-positive bacterium has shown resistance to rifampicin, an antibiotic used for the treatment of $\mathrm{TB}^{29}$ Figure 6 shows the antibacterial activity of Css54 against the growth of $S$. aureus in the presence of isoniazid, rifampicin, pyrazinamide and ethambutol. The mixtures of Css54 $\left(14 \mu \mathrm{g} \mathrm{ml}^{-1}\right)$ with isoniazid, pyrazinamide or ethambutol showed an additive effect. However, the combination of Css54 $\left(14 \mu \mathrm{g} \mathrm{ml}^{-1}\right)$ with rifampicin showed a synergistic effect on the same bacterium. Table 4 summarizes the effects found of the AMPs in combination with the antibiotics used. These data suggest that AMPs could be used in the presence of antibiotics to improve the inhibition of clinical important microorganisms.

\section{DISCUSSION}

Two AMPs named La47 and Css54 were isolated from the spider Lachesana sp. and the scorpion C. s. suffusus, respectively. They were eluted on high percentage of acetonitrile from the reverse phase HPLC separation, indicating an elevated hydrophobic character, which was later confirmed by the large amount of aliphatic aminoacid composition in their primaries structures. Such hydrophobic

Table 4 Effect of the mixtures of AMPs with commercial antibiotics on the growth of bacteria

\begin{tabular}{|c|c|c|c|c|}
\hline \multirow[b]{2}{*}{ Antibiotic } & La47/E. coli & Pin2/S.aureus & & \multirow{2}{*}{$\begin{array}{l}\text { Css54/S.aureus } \\
\text { Effect (FIC index) }\end{array}$} \\
\hline & \multicolumn{2}{|c|}{ Effect (FIC index) } & Antibiotics & \\
\hline Ampicillin & Antagonic (4.3) & Additive (1.3) & Ethambutol & Additive (1.6) \\
\hline Chloramphenicol & Synergic (0.8) & Synergic (0.8) & Pyrazinamide & Additive (1.6) \\
\hline Streptomycin & Synergic (0.4) & Synergic (0.5) & Isoniazid & Additive (1.6) \\
\hline Kanamycin & Synergic (0.4) & Synergic (0.5) & Rifampicin & Synergic (0.9) \\
\hline Novobiocin & Additive (1.3) & Synergic (0.3) & & \\
\hline
\end{tabular}

Abbreviations: AMP, antimicrobial peptide; FIC, fractional inhibitory concentration.

nature is a common characteristic of many pore-forming AMPs, and the hydrophobic character of these peptides is always associated with their cytotrophic activity. Indeed, both peptides La47 and Css54 were toxic to E. coli and S. aureus, but only Css54 was hemolytic. 

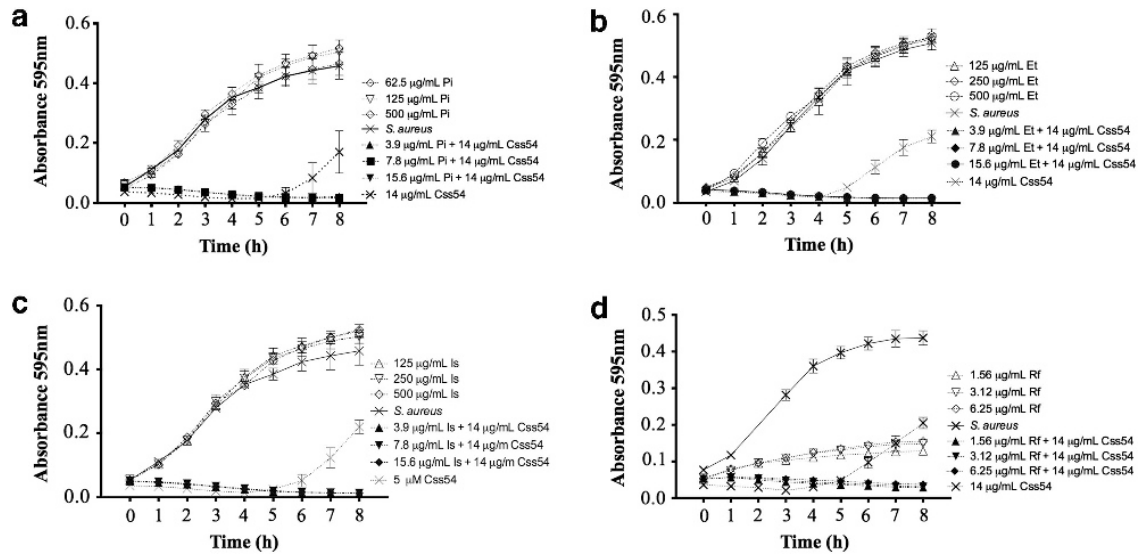

Figure 6 Growth inhibition curves of $S$. aureus in the presence of mixtures of Css54 with antibiotics used to treat tuberculosis. $S$. aureus was grown in the presence of different concentrations of Pyrazinamide (a; 0, 6.5, 125 and $500 \mu \mathrm{g} \mathrm{ml} \mathrm{l}^{-1}$ ), ethambutol (b; 0, 125, 250 and $500 \mu \mathrm{g} \mathrm{ml}{ }^{-1}$ ), isoniazid $(\mathbf{c}$; 0,125 , 250 and $500 \mathrm{gg} \mathrm{ml}^{-1}$ ) and rifampicin (d; $0,1.56,3.12$ and $6.25 \mu \mathrm{g} \mathrm{m} l^{-1}$ ). In addition, $S$. aureus was grown in the presence of combinations of the antimicrobial peptide Css54 (14 $\left.\mathrm{g} \mathrm{ml}^{-1}\right)$ with different concentrations of Pyrazinamide (a; 0, 3.9, 7.8, and 15.6 $\left.\mu \mathrm{ml}^{-1}\right)$, ethambutol (b; 0, 3.9, 7.8, and $15.6 \mu \mathrm{g} \mathrm{ml}^{-1}$ ), isoniazid (c; 0, 3.9, 7.8, and $15.6 \mu \mathrm{g} \mathrm{ml}^{-1}$ ) and rifampicin (d; 0, 1.56, 3.12 and $6.25 \mu \mathrm{g} \mathrm{ml} \mathrm{l}^{-1}$ ).

A previous report showed that the venom of the spider Lachesana tarabaevi contains amphipathic peptides, named latarcins $3 \mathrm{a}$ and $3 \mathrm{~b}$, similar to La47. ${ }^{24}$ Although latarcins $3 \mathrm{a}$ and $3 \mathrm{~b}$ have been described, they have not been chemically synthesized, and their antimicrobial capacity was not tested in the presence of commercial antibiotics. ${ }^{24}$ On the other hand, the AMP Css54 is an unique peptide having low identity to AMPs obtained from the skin of amphibian species ${ }^{26,27,30}$ and from the venoms of the Hymenoptera. ${ }^{31}$ However, it has a low identity $(41 \%)$ to the AMP Pin2 obtained from the venom of the related scorpion Pandinus imperator ${ }^{10}$ but a higher identity (60\%) to ponericins from the venom of the ant Pachycondyla goeldii. ${ }^{25}$ From the molecular diversity point of view, the slight identity of Pin2, Ponericins and Css54 support the hypothesis that they have a common peptide ancestor, but more specifically it might point towards the evolution of a particular type of venom gland used for protection and/or weapon in the phylum Arthropoda. Also, it is remarkable in all of these peptides, including the AMPs from amphibians, the conservation of the motif KXXP where the double $\mathrm{X}$ represents two hydrophobic residues such as $\mathrm{I}, \mathrm{L}, \mathrm{V}$ or $\mathrm{F}$, and also a second motif represented by the basic dipeptide $\mathrm{KK}$ at the C-terminal of these AMPs (Table 2). These two motifs seem to be well represented in invertebrates and vertebrates and may be an interesting indication to design de novo AMPs.

Furthermore, the primary structure of La47 and Css54 are typically of those cationic peptides mainly composed of lysine amino-acid residues and hydrophobic peptides such as alanine, isoleucine and leucine that give a particular $\alpha$-helix structure to them. Css54 most probably contains two $\alpha$-helices separated by a characteristic Pro residue (Pro14), which is a $\alpha$-helix breaker amino acid. Several other $\alpha$-helical AMPs present similar structural features like the antimicrobial cecropin A where the Pro23 residue characterizes a flexible region, ${ }^{32}$ and sarcotoxin A where two residues, Gly24 and Ile27, characterize a hinge region. ${ }^{33}$

Both La47 and Css54 were chemically synthesized, and each had similar retention time, under reverse phase HPLC, compared with that of their native counterparts. Additionally, the molecular masses of the two synthetic peptides La47 and Css54 were also confirmed by mass spectrometry analysis. These data confirmed indirectly their correct structure. The antimicrobial activities of La47 and Css54 were correlated to its hemolytic activity. In this respect, it is important to notice that the higher the antimicrobial activity of an amphipathic peptide is, higher is its hemolytic activity, which is also correlated to its hydrophobicity. For example lycotoxins and Pin2 are more hydrophobic and hemolytic than magainins. ${ }^{10,14}$ Similarly, La47 is not hemolytic to human red blood cells up to $80 \mu \mathrm{g} \mathrm{ml}^{-1}$ but the hydrophobic Pin 2 and Css54 produce 80 to $100 \%$ hemolysis of human blood cells at the same concentration, respectively. Likewise, it has been noticed that hydrophobic peptides having both hemolytic and antimicrobial activities are more effective in permeabilizing lipid vesicles. ${ }^{34}$ In this respect, there is a positive correlation between hemolytic and antibacterial activity of AMPs. Therefore, hemolytic peptides are stronger antibiotics, but also they are cytotoxic to eukaryotic cells. Here the point is that we foresee the application of these mixtures only at topical applications sites for the moment, in order to treat bacterial infections restricted to epithelial tissues. In this regards we should note that epithelial cells are quite resistant to cytolytic agents than other cells, such as erythrocytes. ${ }^{7,9}$

Since AMPs act by pore-formation, it was an interesting idea to test them in the presence of low molecular weight commercial antibiotics. Remarkably, mixtures of Css54 and commercial antibiotics increase the bacteriostatic or bactericidal efficiency on $S$. aureus. This phenomenon may be related to the fact that AMPs permeate the lipidic cell membranes and help to the diffusion of low molecular weight antibiotics. Interestingly, the most effective antibiotics were those that act on protein structures in the bacterial cytoplasm such as the ribosomal subunit $30 \mathrm{~S}$ (kanamycin and streptomycin). These data indirectly show that AMPs most probably form cell membrane gaps that permit the exchange of organic material from the outside to the inside of the bacterial cell and vice versa.

Since the number of amino-acid residues of La47, Css54 and Pin2 are 20, 25 and 24, respectively, the length of these peptides is expected to be $\sim 36 \AA$ ( $1.5 \AA$ per residue). Therefore, they are large enough to span through the bacterial cell membranes forming pore-like structures. Belokoneva et al. ${ }^{18}$ reported that pores created by $P$ in 2 in artificial vesicles permit perfusing of calcein $(623 \mathrm{Da})$ freely because the pore size radii induced by Pin 2 could expand from 1.8 to $5 \mathrm{~nm}$ in a peptide-lipid ratio-dependent manner. Therefore, because of their similar size, La47 and Css54 are expected to generate membrane pores as Pin2 allowing the passage of low molecular weight antibiotics. 
In this respect, recent discoveries have shown that iron uptake systems in bacteria and fungi are suitable targets for developing siderophore-drug conjugates as 'Trojan Horse' drug delivery agents. Therefore, AMP conjugates could be used as 'Trojan Horse' antibiotics in a similar way as salmycins, a series of desferridanoxamine-antibiotic conjugates tested for microbial growth inhibition assays. Furthermore, AMPs could be coupled to specific outer surface antibodies or to a specific ligand for bacterial pathogen receptors to develop novel therapeutic agents. ${ }^{35}$ In this way, synergy or additive interactions would allow low doses of individual commercial antibiotics to be used more effectively against pathogenic bacteria. Since AMPs has a high efficiency in killing pathogens and many synthetic analogs and synthetic derivatives have successfully been developed to improve their biological activity as well as the capability for being accepted as new substitutes for conventional pesticides and antibiotics, the use of AMPs could have a promising future for extensive applications in medicine, agriculture, aquaculture, animal husbandry and food industry. ${ }^{20}$

In conclusion, the most significant result of the experiments described here is the fact that combination of AMPs with commercially available antibiotics certainly increases the efficiency of the mixtures in preventing microbial infections and bacterial growth. These data set the basis for development and formulation of adequate mixtures of both AMPs and known antibiotics for the control of diseases.

\section{ACKNOWLEDGEMENTS}

The mass spectrometry determination conducted by Dr Fernando Zamudio is greatly acknowledged. We also acknowledge QBP Ma. Rocio Patiño Maya from Instituto de Química-UNAM for CD spectra data acquisition. The patent filling advice from MSc Martin Patiño and MBA Mario Trejo is also greatly acknowledged. Francia García was recipient of an MSc scholarship (\#215024) from CONACyT. This work was partially supported by grants from Laboratorios Silanes SA de CV and CONACyT 153606.

1 Jovetic, S., Zhu, Y., Marcone, G. L., Marinelli, F. \& Tramper, J. Beta-Lactam and glycopeptide antibiotics: first and last line of defense? Trends. Biotechnol. 28, 596-604 (2010).

2 Pallecchi, L., Bartoloni, A., Paradisi, F. \& Rossolini, G. M. Antibiotic resistance in the absence of antimicrobial use: mechanisms and implications. Expert. Rev. Anti. Infect Ther. 6, 725-732 (2008)

3 Walsh, C. Where will new antibiotics come from? Nat. Rev. Microbiol. 1, 65-70 (2003).

4 Lambert, R. J. \& Lambert, R. A model for the efficacy of combined inhibitors. J. Appl. Microbiol. 95, 734-743 (2003).

5 Linden, P. K. Treatment options for vancomycin-resistant enterococcal infections. Drugs 62, 425-441 (2002).

6 Maróti, G., Kereszt, A., Kondorosi, E. \& Mergaert, P. Natural roles of antimicrobial peptides in microbes, plants and animals. Res. Microbiol. 162, 363-374 (2011).

7 Brogden, N. K. \& Brogden, K. A. Will new generations of modified antimicrobial peptides improve their potential as pharmaceuticals? Int. J Antimicrob. Agents 38, 217-225 (2011).

8 Steinstraesser, L., Kraneburg, U., Jacobsen, F. \& Al-Benna, S. Host defense peptides and their antimicrobial-immunomodulatory duality. Immunobiology 216, 322-333 (2011).

9 Villegas, E. \& Corzo, G. Pore-forming peptides from spiders. Toxin Rev. 24, 345-357 (2005)
10 Corzo, G. et al. Characterization of unique amphipathic antimicrobial peptides from venom of the scorpion Pandinus imperator. Biochem. J 359, 35-45 (2001).

11 Kuhn-Nentwig, L. Antimicrobial and cytolytic peptides of venomous arthropods. Cell Mol. Life Sci. 60, 2651-2668 (2003).

12 Ramirez-Carreto, S. et al. Gene cloning and functional characterization of four novel antimicrobial-like peptides from scorpions of the family Vaejovidae Peptides. Peptides 34, 290-295 (2012)

13 Torres-Larios, A., Gurrola, G. B., Zamudio, F. Z. \& Possani, L. D. Hadrurin, a new antimicrobial peptide from the venom of the scorpion Hadrurus aztecus. Eur. J. Biochem. 267, 5023-5031 (2000).

14 Yan, L. \& Adams, M. E. Lycotoxins, antimicrobial peptides from venom of the wolf spider Lycosa carolinensis. J. Biol. Chem. 273, 2059-2066 (1998).

15 Zeng, X. C., Corzo, G. \& Hahin, R. Scorpion venom peptides without disulfide bridges. IUBMB Life 57, 13-21 (2005).

16 Conde, R., Zamudio, F. Z., Rodriguez, M. H. \& Possani, L. D. Scorpine, an anti-malaria and anti-bacterial agent purified from scorpion venom. FEBS Lett 471, 165-168 (2000).

17 Corzo, G. et al. Oxyopinins, large amphipathic peptides isolated from the venom of the wolf spider Oxyopes kitabensis with cytolytic properties and positive insecticidal cooperativity with spider neurotoxins. J. Biol. Chem. 277, 23627-23637 (2002).

18 Belokoneva, O. S. et al. Pore formation of phospholipid membranes by the action of two hemolytic arachnid peptides of different size. Biochim. Biophys. Acta. 1664, 182-188 (2004).

19 Belokoneva, O. S., Villegas, E., Corzo, G., Dai, L. \& Nakajima, T. The hemolytic activity of six arachnid cationic peptides is affected by the phosphatidylcholine-to-sphingomyelin ratio in lipid bilayers. Biochim. Biophys. Acta. 1617, 22-30 (2003).

20 Keymanesh, K., Soltani, S. \& Sardari, S. Application of antimicrobial peptides in agriculture and food industry. World J Microbiol Biotechnol. 25, 933-944 (2009).

21 Sonnichsen, F. D., Van Eyk, J. E., Hodges, R. S. \& Sykes, B. D. Effect of trifluoroethanol on protein secondary structure: an NMR and CD study using a synthetic actin peptide. Biochemistry 31, 8790-8798 (1992).

22 Eliopoulos, G. M. \& Moellering, R. C. Antimicrobial combinations. Antibiotics in laboratory medicine (ed Lorian, V.) 3rd edn. BaltimoreThe Williams \& Wilkins Co (1991).

23 White, R. L., Burgess, D. S., Manduru, M. \& Bosso, J. A. Comparison of three different in vitro methods of detecting synergy: time-kill, checkerboard, and E test. Antimicrob. Agents Chemother. 40, 1914-1918 (1996).

24 Kozlov, S. A. et al. Latarcins, antimicrobial and cytolytic peptides from the venom of the spider Lachesana tarabaevi (Zodariidae) that exemplify biomolecular diversity. J. Biol. Chem. 281, 20983-20992 (2006).

25 Orivel, J. et al. Ponericins, new antibacterial and insecticidal peptides from the venom of the ant Pachycondyla goeldii. J. Biol. Chem. 276, 17823-17829 (2001).

26 Park, J. M., Jung, J. E. \& Lee, B. J. Antimicrobial peptides from the skin of a Korean frog. Rana rugosa. Biochem. Biophys. Res. Commun. 205, 948-954 (1994).

27 Morikawa, N., Hagiwara, K. \& Nakajima, T. Brevinin-1 and -2, unique antimicrobial peptides from the skin of the frog, Rana brevipoda porsa. Biochem. Biophys. Res. Commun. 189, 184-190 (1992).

28 Zasloff, M. Antimicrobial peptides of multicellular organisms. Nature 415, 389-395 (2002).

$29 \mathrm{Liu}$, Y. et al. Selection of rifampicin-resistant Staphylococcus aureus during tuberculosis therapy: concurrent bacterial eradication and acquisition of resistance. J. Antimicrob. Chemother. 56, 1172-1175 (2005).

30 Goraya, J., Knoop, F. C. \& Conlon, J. M. Ranateurin 1T: an antimicrobial peptide isolated from the skin of the frog, Rana temporaria. Peptides 20, 159-163 (1999).

31 Nakajima, T. Pharmacological biochemistry of Vespid venoms. In Venoms of Hymenoptera (ed Piek, T.) (Academic Press, London), 1986.

32 Steiner, H., Andreu, D. \& Merrifield, R. B. Binding and action of cecropin and cecropin analogues: antibacterial peptides from insects. Biochim. Biophys. Acta. 939, 260-266 (1988)

33 Iwai, H., Nakajima, Y., Natori, S., Arata, Y. \& Shimada, I. Solution conformation of an antibacterial peptide, sarcotoxin IA, as determined by ${ }^{1} \mathrm{H}-\mathrm{NMR}$. Eur. J. Biochem. 217 639-644 (1993)

34 Sitaram, N. \& Nagaraj, R. Interaction of antimicrobial peptides with biological and model membranes: structural and charge requirements for activity. Biochim. Biophys. Acta. 1462, 29-54 (1999).

35 Wencewicz, T. A., Möllmann, U., Long, T. E. \& Miller, M. J. Is drug release necessary for antimicrobial activity of siderophore-drug conjugates Syntheses and biological studies of the naturally occurring salmycin "Trojan Horse" antibiotics and synthetic desferridanoxamine-antibiotic conjugates. Biometals 22, 633-648 (2009). 